\title{
Ultrasound effect on radiation damages in boron implanted silicon
}

\author{
B. Romanjuk, D. Krüger*, V. Melnik, V. Popov, Ya. Olikh, V. Soroka**, O. Oberemok \\ Institute of Semiconductor Physics of NASU, 45, prospect Nauki, 03028, Kyiv, Ukraine. Tel/Fax: (38044)265-5724. \\ E-mail: romb@isp.kiev.ua. \\ *Institute for Semiconductor Physics, W. - Korsing - Str. 2, 15230 Frankfurt (Oder), Germany. Tel.: (49335)5625-326. \\ Fax,: (49335) 5625-300
}

\begin{abstract}
The radiation defect distribution in boron implanted Si with and without ultrasound (US) treatment have been investigated. Obtained results have shown the significant influence of the in situ US treatment on the defect formation. The defect concentration decreases both in the as-implanted and post-annealed samples, implanted with US-treatment. The physical model of this effect, connecting it with stimulated diffusion of silicon interstitials under the US treatment have been proposed.
\end{abstract}

Keywords: ion implantation, silicon, Rutherford backscattering spectroscopy, $p$ - $n$ junction, boron, microelectronics, radiation defects, diffusion.

Paper received 17.12.99; revised manuscript received 04.01.00; accepted for publication 06.01.00.

\section{Introduction}

Ion implanted boron is commonly employed as the $p$ type dopant in semiconductor devices. But $p^{+}-n$ shallow junction formation by boron implantation is complicated by the two phenomena: channelling effect, and anomalous diffusion [1]. Channelling effect can be suppressed by using Si preamorphization with $\mathrm{Si}^{+}$or $\mathrm{Ge}^{+}$implantation prior to implantation doping [2]. The problem is that preamorphization leads, after annealing, to the formation of high structural defect densities near former crystalline/amorphous interface. These defects are called endof-range defects and they are present in all the materials by high-dose ion implantation in which a continuous amorphous layer is formed [3]. High-dose B implantation for sources and drains are required. After implantation the annealing steps are required to repair the damage in the silicon lattice and to activate the dopant. The presence of any residual damage after annealing may have a significant effect on the electrical properties of the implanted layer [4].

The effective amorphization threshold damage density is a strong function of both ion mass and wafer temperature during implantation. The threshold damage density increases as the mass of the ion decreases. On the other hand, heating of the wafer results in increased of point defects recombination, which decreases amorphization. Part of point defects recombines during implantation. Competition between generation and recombination of point defects results in a wide amorphous region. The nature of the residual displacement depends upon factor such as the incident ion mass, energy, dose rate, temperature, state of surface and substrate orientation, presence of additional factors, as the electrical or mechanical fields [5].

It has been suggested that ultrasonic (US) waves, propagating through the semiconductor can effect the generation of point defects and dissociation of defect complexes [6]. In this connection it seems to be perspective to use an in-situ ultrasonic treatments during the implantation process, when the target atoms are in an exited state, and defect complexes are unstable.

The present study aims to investigate the ultrasounddependent amorphization and electrical activation of boron impurity during ion implantation in silicon to determine in more details the effect of point defect outdiffusion on the kinetics of amorphization process. We show that the defect density in the surface layer, and the thickness of the amorphous layer depends on the US treatment. We discuss the results in frames of the point defect separation and out-difiusion model. 


\section{B. Romanjuk et al.: Ultrasound effect on radiation damages in boron implanted silicon...}

\section{Experiment}

Boron-doped $10 \mathrm{Ohm} \cdot \mathrm{cm}$ Czochralsky-grown (Cz-Si) Si wafers (100) were mounted inside the implantation chamber onto piezoelectric transducers via special acoustics binder. Low-amplitude US vibrations were generated in the wafer by means of the transducer, operating in resonance vibration mode. We varied the basic resonance frequency from $600 \mathrm{kHz}$ to $7 \mathrm{MHz}$. The amplitude of acoustic strain did not exceed $10 \mathrm{~N} / \mathrm{cm}^{2}$ corresponding to an acoustic power of $1 \mathrm{~W} / \mathrm{cm}^{2}$. For implantation we used $\mathrm{B}^{+}$ions with energies of $50 \mathrm{keV}$ and doses of $1 \cdot 10^{16} \mathrm{~cm}^{-2}$. The ion flux was $3 \cdot 10^{12}$ ions $/ \mathrm{cm}^{2}$. The part of samples were implanted without US-treatment. The rapid thermal annealing $\left(900^{\circ} \mathrm{C}, 30 \mathrm{~s}\right)$ was carried out after ion implantation.

Rutherford backscattering spectrometry (RBS) combined with channeling was employed for damage studies. The depth profile of the lattice disorder was determined from $<100>$ channeling of $1.5 \mathrm{MeV} \alpha$-particles. The approach described in [7] has been used. This approach uses an iterative procedure to determine the amount of dechanneled particles. The method is based on the next assumptions: all the displaced atoms are distributed randomly and can interact with the channeled particles; the critical angle of channeling is unchanged by the introduction of disorder, the flux of channeled particles is distributed uniformly within the channels, dechanneled particles are not scattered back into channels.

The disorder concentration $N_{D}(x)$ is given by

$N_{D}(x)=N\left[\chi_{R}(x)-\chi(\mathrm{x})\right] /\left[1-\left[\chi_{R}(x)\right]\right.$,

where $N$ is bulk density (in atoms per cubic centimeter); $\chi(x)$ is the normalized yield (the ratio of aligned to random yield at the energy $E$, where the disorder is evaluated); $\chi_{R}(x)$ is the fraction of dechanneled particles (the random component of the aligned beam) and $\left[1-\left[\chi_{R}(x)\right]\right.$ is the fraction of channeled particles, that can interact with $N_{D}(x)$ displaced atoms.

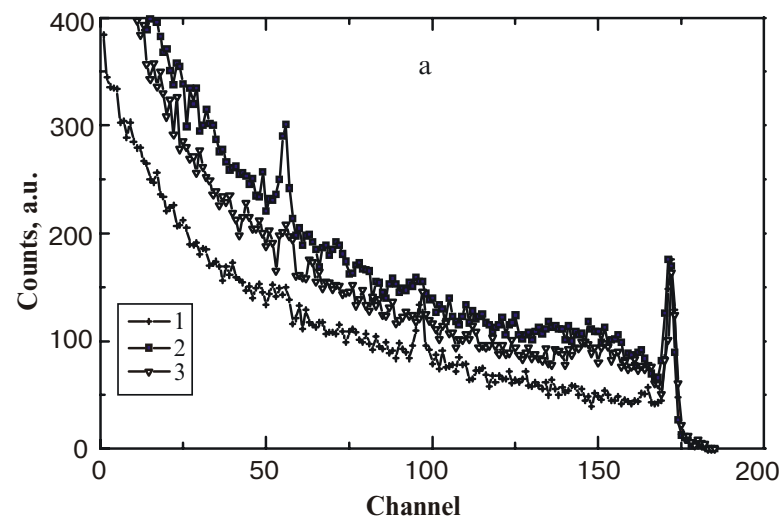

The dechanneled fraction is approximated by

$\chi_{R}(x)=\left[\chi_{\nu}(x)+\left[1-\chi_{V}(x)\right] P\left(x, \phi_{1 / 2}\right)\right.$,

where $\chi_{V}(x)$ is the aligned yield from a virgin crystal and $P\left(x, \phi_{1 / 2}\right)$ is the probability that channeled particles are dechanneled by defects between the surface and depth.

The probability $P\left(x, \phi_{1 / 2}\right)$ is given by

$P\left(x, \phi_{1 / 2}\right)=\sigma_{D}\left(\phi_{1 / 2}\right) N_{D}(x) x$

where $\sigma_{D}\left(\phi_{1 / 2}\right)$ is the cross section for atoms to deflect particles though angles equal to or greater than channeling critical angles $\phi_{1 / 2}$. To estimate the cross section $\sigma_{D}\left(\phi_{1 / 2}\right)$ one uses the Rutherford differential scattering cross section and integrates from $\phi_{1 / 2}$ to $\infty$. It should also be noted that different procedures to determine the beam dechanneled fraction has been used in the work [8], where damage depth profile of B-implanted single crystals of silicon has been studied too.

\section{Results}

Fig. la presents aligned backscattering spectra for implanted samples with and without US treatment. For comparison the aligned spectrum from the virgin crystal (without implantation procedure) is also given. The backscattenng yield due to $\mathrm{Si}$ displaced atoms increases in the range of 125-160 channels, the more pronounced for control sample. US treatment results in decreasing of backscattering yield in all the range of channels. These yields were converted to a concentration of displaced atoms and it is presented in this Fig. The concentration of displaced atoms is lower in the samples treated by US with the frequency of $7 \mathrm{MHz}$.

Fig. 1b shows RBS-c spectra illustrating the effect of US treatment for the annealed samples. For the sample annealing the dechanneled ion yield substantially decreases in the region of implanted boron distribution, the

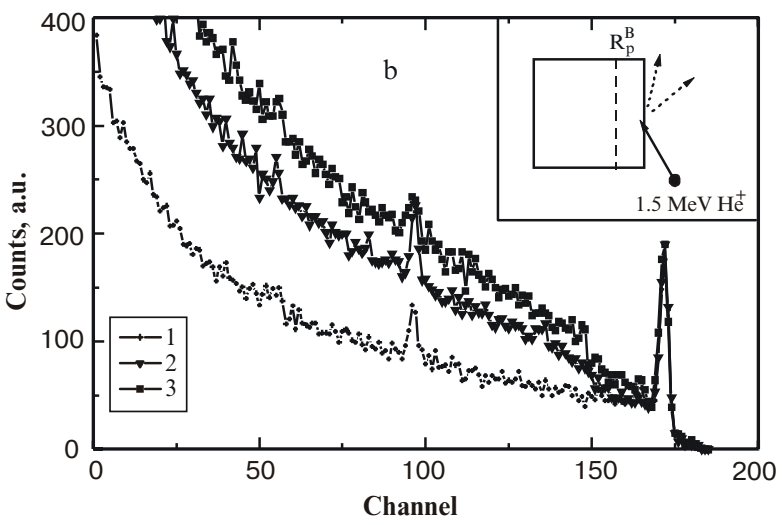

Fig. 1. Aligned RBS spectra of the $50 \mathrm{keV} \mathrm{B}{ }^{+}$-implanted Si wafers without US treatment (2), with US treatment at $7 \mathrm{MHz}$ (3) compared with $<100>$ aligned spectrum for unimplanted $\mathrm{Si}(1)$, before (a) and after sample annealing (b). Insert: schematic presentation of the RBS experiment. 


\section{B. Romanjuk et al.: Ultrasound effect on radiation damages in boron implanted silicon...}

effect is stronger for the samples with US treatment (7 MHz).

Distribution of defects that cause ion dechanneling in the dependence on the distance from sample surface is shown in Fig. 2. Random stopping power for the incoming trajectory is used to obtain the depth scale from the channeling spectra because the damage is shallow in the present case.

In the implanted samples the profile of displaced ion distribution is close to that, calculated by TRIM, with the exception of higher concentration of dechanneled centers. Concentration of displaced atoms in the samples implanted under US action (especially for $f=7 \mathrm{MHz}$ ) is substantially less. Moreover, in the samples with US treatment after annealing, the profile of defect distribution is displaced to the sample volume.

Displaced atoms are distributed in larger distance from the surface. The defect concentration in the samples implanted under US action is lower, especially in the region of boron atom distribution, within the range of 0 to $200 \mathrm{~nm}$.

\section{Discussion}

Implantation by light ions leads to generation of point defects and their complexes. Under RT the amorphous layer does not arise [1]. Defects are distributed in the region close to the distribution of boron ions. During the implantation, when dominant type of defects are the single ones and not the disordered regions, as it takes place for heavy ion, the main part of defects recombine within the short time $\sim 10^{-11} \mathrm{~s}\left(\mathrm{I}+\mathrm{V} \rightarrow \mathrm{Si}_{\mathrm{i}}\right)$. Interstitial silicon de-

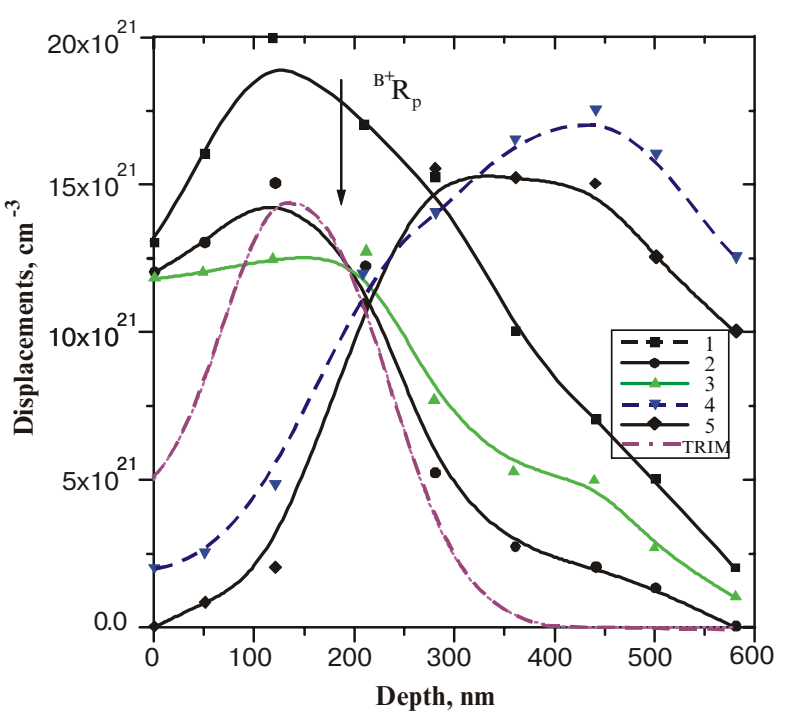

Fig. 2. Depth distribution of the displaced atoms for the $\mathrm{B}^{+}$asimplanted Si without US treatment (1), with US treatment at 2 $\mathrm{MHz}$ (2), $7 \mathrm{MHz}$ (3), and after annealing of the samples, implanted without US (4) and with US (5). Dashed line presents the defect distribution calculated by TRIM. fects and interstitial boron atoms increase the yield of dechanneled particles.

Ultrasonic irradiation may act upon the quasi-chemical reaction proceeding $\left(\mathrm{B}_{\mathrm{i}}+\mathrm{V} \rightarrow \mathrm{B}_{\mathrm{s}} ; \mathrm{V}+\mathrm{I} \rightarrow \mathrm{Si}_{\mathrm{s}}\right)$, as well as upon the point defect diffusion. As the calculations [9] have shown, influence of US on diffusion depends on type of diffusing atom. For light atoms the effects of localization may be observed, while for heavy atoms the effects of diffusion acceleration take place.

We have shown that US vibration accelerates amorphization of Si under implantation by $\mathrm{Ar}^{+}$ions. It is connected with spatial separation of point defects. Diffusion of interstitial atoms is accelerated, while vacancies assemble themselves to the more complex multi-vacancy agglomerations. As a result, the point defect recombination is lowered, and the more intensive defect accumulation up to the critical amorphization value occurred.

The similar mechanism acts also for the case, when more light boron ions are implanted. Interstitial atoms diffuse inside the crystal under the action of ultrasound. At the same time proceeding the $\mathrm{B}_{\mathrm{i}}+\mathrm{V} \rightarrow \mathrm{B}_{\mathrm{s}}$ reaction is stimulated, since vacancy concentration in the region of boron distribution increases. As a result, we have an increase of dechanneled particle concentration. Our investigations confirm these speculations.

Implantation damage induces extended defects such as dislocation loops or $\{311\}$ defects in the damage-region during thermal annealing. The $\{311\}$ defects consist from condensates of interstitials, forming five and seven-membered rings. These defects increase greatly in size during rapid thermal annealing. The size of these defects is $\sim 10 \mathrm{~nm}$ and concentration is near $10^{12} \mathrm{~cm}^{-2}$. The dechanneling probability due to the dislocation loops which arise after implanted sample annealing, increases [8]. For implanted samples with US-treatment, the concentration of these defects is lower, because part of more mobile interstitials diffuses into bulk of silicon. We must note that US treatment stimulates the boron solubility and suppresses boron clusterization. It is shown from the dechanneling center distribution in the reference and US treated implanted samples after annealing in the region of boron distribution. The redistribution of vacancies towards surface, which act as sink of boron atoms, depends on the US frequency.

\section{Conclusions}

Our investigations of the defect distribution into boron implanted Si with and without US treatment have shown the significant influence of US on the defect formation. The defect concentration decreases both in the as-implanted and post-annealed samples, implanted with US treatment. We suggest that this effect is connected with stimulated diffusion of silicon interstitials under the US treatment. 


\section{B. Romanjuk et al.: Ultrasound effect on radiation damages in boron implanted silicon...}

\section{Acnowlegements}

We would like to thank Prof . V.Litovchenko and Prof. A. Ourmazd for helpful discussion.

\section{References}

1. P.A. Stolk, H.J. Cossmann, D.J. Euglesham et al., Physical mechanisms of transient enhanced dopant diffusion in ionimplanted Si // J. Appl. Phys. 81(9), pp. 6031-6050(1997)

2. A. Ferriero, B. Biasse, A.M. Papon et al., Formation of the shallow $\mathrm{p}^{+}$-n junctions by dual $\mathrm{Ge}^{+} / \mathrm{B}^{+}$implantation // Nucl. Instr. \&Meth. in Phys. Res., B39 , pp. 413-416 (1989)

3. C. Bonafos, A. Claverie, D. Aiguier et al., The effect of the boron doping level on the thermal behavior of end-of-range defects in silicon // Appl. Phys. Lett., 71 (3), pp. 365-367 (1997)
4. W. Vandervorst, D.C. Houghton, F.R. Shepherd et al., Residual damage in $\mathrm{B}^{+}$and $\mathrm{BFi}^{+}$-implanted $\mathrm{Si} / /$ Can. J. Phys., 63 (2), pp. 863-869 (1985)

5. J. Thornton, R.P. Webb, I.H. Wilson, K.C. Paus, Predicted dose, energy and implantation temperature effects on the residual disorder following the annealing of pre-amorphized silicon // Semicond. Sci. \& Technol., 3 (2), pp. 281-285 (1988)

6. I.V.Ostrovskii and V.N.Lysenko, Ultrasonic generation of point defects in CdS // Sov. Phys. Solid State, 24 (5), pp. 682-683 (1982).

7. W.K. Chu, J.W. Mayer, M.A. Nicolet, Backscattering Spectromefry, N.Y. (1977)

8. M. Vos, D.0. Boerma, P.J.M. Smulders, S. Oosterhoff, Defect and dopant depth profiles in boron-implanted silicon studied with channeling and nuclear reaction analysis //Nucl. Instr. \& Meth. in Phys. Res, B17, pp. 234-241 (1986)

9. I.V.Pavlovich. Enhanced diffusion of impurities and defects in crystals in conditions of ultrasonic treatment // Phys. Stat. Sol., B180 (1), pp. 97-105 (1993). 